\title{
Training in metrology in the National Agricultural Technology Institute (INTA)
}

\author{
Gomez, Mario ${ }^{1}$ - Kremer, Raul - Slepetis, Cristina \\ ${ }^{1}$ Laboratory of Proficiency Tests, Standards and Metrology - Process and Quality Management - INTA \\ Av. Chile 460, Buenos Aires City, Argentina
}

Key Words: calibration, training, government agency, measurement

\section{INTRODUCTION}

The National Institute of Agricultural Technology is a decentralized government agency with operational and financial autonomy, under the dependency of the Ministry of Agroindustry. Since its creation in 1956 INTA has researched and given technological innovation solutions for the value chains, regions and territories in order to improve the competitiveness and sustainable of rural development in Argentina. INTA has several agencies in each province of the country, with more 9000 employees.

INTA promotes the research, development and extension for giving solutions the growing demands of the agricultural sector. In its Institutional Strategic Plan 2005-2015 and 2015-2025 adopts international ISO standards models to establish its Quality Management System (QMS), demonstrating its commitment to improvement and pursuing the excellence in the services provided. INTA needs to ensure the results of measurements made by its laboratories, as well as the results of the teams involved in support research services. Thus, the laboratories of the institution must demonstrate that they generate reliable and valid results. Consequently, one of the requirements is that every equipment use that has a significant effect on the accuracy or validity of the results must be calibrated, verified and maintained regularly to have assurance in the results given.

However, until the creation of the metrological project, INTA didn't have a systematic institutionalized calibration or verification procedure for critical laboratory and agencies equipment. Most of the staff wasn't trained to perform these tasks neither to understand calibration documents or the impact of the results of calibration. This task was usually outsource with high costs and logistical problems (delay in service provision and eventually in its quality).

Given this scenario, in 2008 INTA began working in the first draft for the Measurement Management System (SGM) based on six pillars:

- Development of tools for calibration or calibration software

- Formation and staff training

- Acquisition, maintenance and calibration of reference equipment

- Development of INTA's Central Metrology Laboratory

- Participation in national and international proficiency tests

- Implementation of a quality system based on ISO/IEC17025 to be evaluate by the Argentine Accreditation Body (OAA) in order to demonstrate technical competence

A great impetus to the creation of SGM was the approval of INTA -BID project "Investment in instrumental to calibrate and verify the Institutional critical equipment according to the International Units 
System (SI) and Staff Training". This enabled the purchase of reference equipment and formation and staff training.

The aim of this work is to develop the main aspects of the formation and training of staff Metrology under the SGM project.

\section{DEVELOPMENT}

To achieve compliance with the objective of SGM two action plans were establish. First, the design of the calibration software that allows calibrating in a few minutes without the need of making any staff calculation was design. The software was designed in Excel and was based on internationally accepted calibration procedures (1). Chronologically the development of the software was:

- 2005: balance calibration software

- 2009: volumetric calibration software

- 2011: thermometers calibration software

- 2015: weights calibration software

The second action plan was the communication, formation and staff training in the basic aspects of metrology, in the use of the calibration software, in the interpretation of results and their impact on the respective measurement.

Once trained and after performing correctly a calibration, the person is accepted as an authorized calibration agent for that magnitude. The authorized calibration agents can perform calibrations or verifications in INTA's agencies around the country.

\section{MATERIALS AND METHODS}

Since its creation, the SGM has registered and stored the information on formation and trainings offered. The information of the period 2005-2016 was grouped by type (classroom or virtual) and classified by topic or magnitude (2).

It also has been registered the number of authorized agents for each magnitude and the amount of calibrations performed during that period.

Since December 2012 the management of the courses are certified under ISO 9001 with IRAM.

\section{RESULTS AND DISCUSSION}

In the period 2005-2016, the SGM held 17 courses. In Table 1 the same are presented ordered by methodology and subject. $51 \%$ of the trainings were given in a classroom and the remaining $49 \%$ as a distance course. In the period, 348 were formed in the subject.

If we evaluate the effectiveness of formation and training done by measuring the amount of authorized agents during this period, we observe that nowadays we have an overall the 102 agents, 56 allowed to calibrated balances, 36 volumetric items and 10 thermometer (Fig.2).

If we analyze the number of calibrations performed in the period 2011 to 2016 we observe in Fig. 3 a significant increase in the number of calibrations performed in the last period. In relation to the above, it has also increased the saving of government resources from \$12,990 (dollars) in 2011 to \$ 85,216 (dollars) in 2016. In the 2011-2016 period, 2003 in-house calibrations were carried out, representing a saving of INTA resources of $\$ 214,317$ (dollars). (Fig. 4) 
Table 1. Number of courses organized by the SGM and number of trained agents by subject in the period 2005-2016

\begin{tabular}{|c|c|c|c|c|c|c|}
\hline \multirow[b]{2}{*}{ Ref } & \multirow{2}{*}{$\begin{array}{l}\text { Number of } \\
\text { students }\end{array}$} & \multicolumn{2}{|c|}{ Mode } & \multicolumn{3}{|c|}{ Magnitude } \\
\hline & & Classroom & Virtual & $\begin{array}{c}\text { Mass } \\
\text { (balances) }\end{array}$ & Volume & Temperature \\
\hline Imyza 2005 & 26 & * & & * & & \\
\hline PROCADIS 1 & 45 & & * & * & & \\
\hline PROCADIS 2 & 19 & & * & * & & \\
\hline PROCADIS 3 & 29 & & * & * & & \\
\hline CA1105 & 19 & * & & * & * & \\
\hline CA1111 & 15 & * & & * & * & \\
\hline CA1211 & 14 & * & & * & * & \\
\hline CA1222 & 19 & * & & & & * \\
\hline CA1312 & 20 & * & & * & * & * \\
\hline CA1318 & 27 & * & & * & * & \\
\hline CA1403 & 9 & & * & * & & \\
\hline CA1404 & 16 & & * & * & * & * \\
\hline CA1405 & 16 & * & & & & \\
\hline CA1501 & 15 & * & & * & * & * \\
\hline CA1503 & 22 & & * & * & * & * \\
\hline CA1505 & 28 & * & & * & * & \\
\hline CA1602 & 9 & & * & & * & \\
\hline TOTAL & 348 & 10 & 7 & 14 & 10 & 5 \\
\hline
\end{tabular}




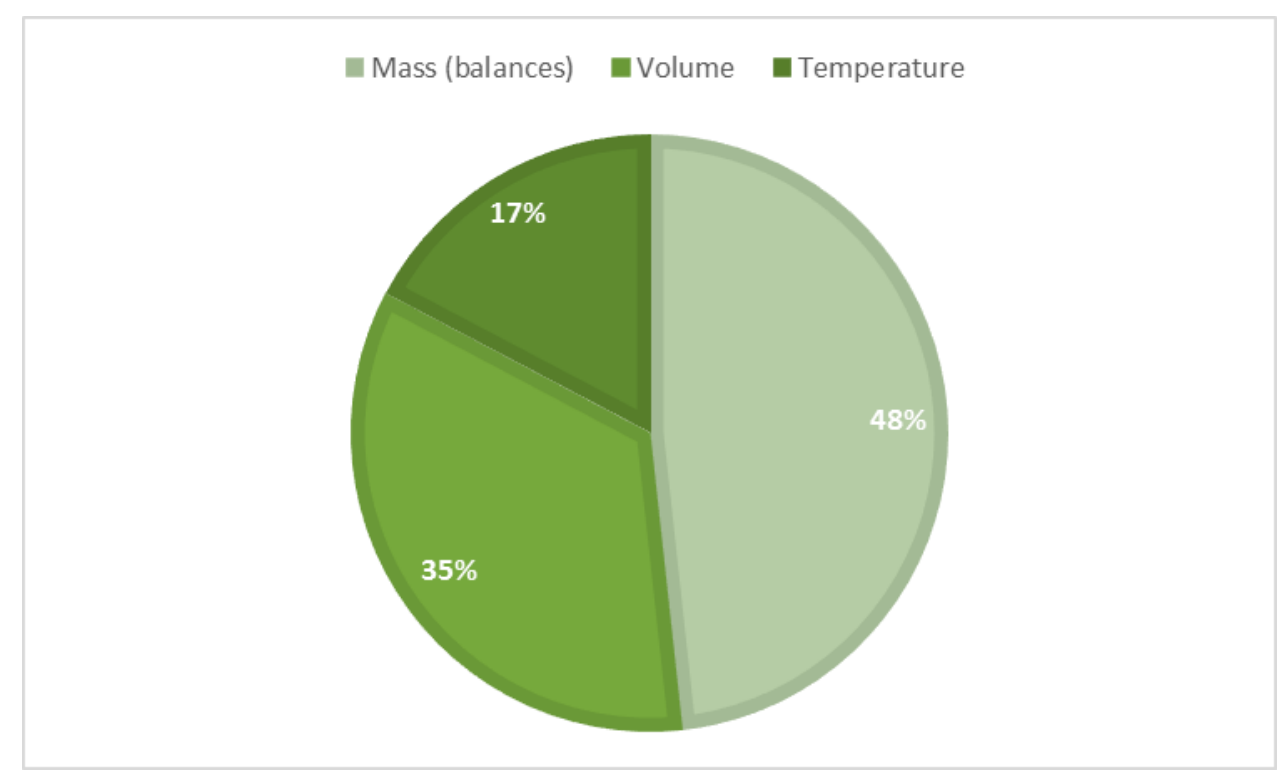

Fig. 1. Distribution of courses and calibration training for each magnitude in the period $2005-2016$

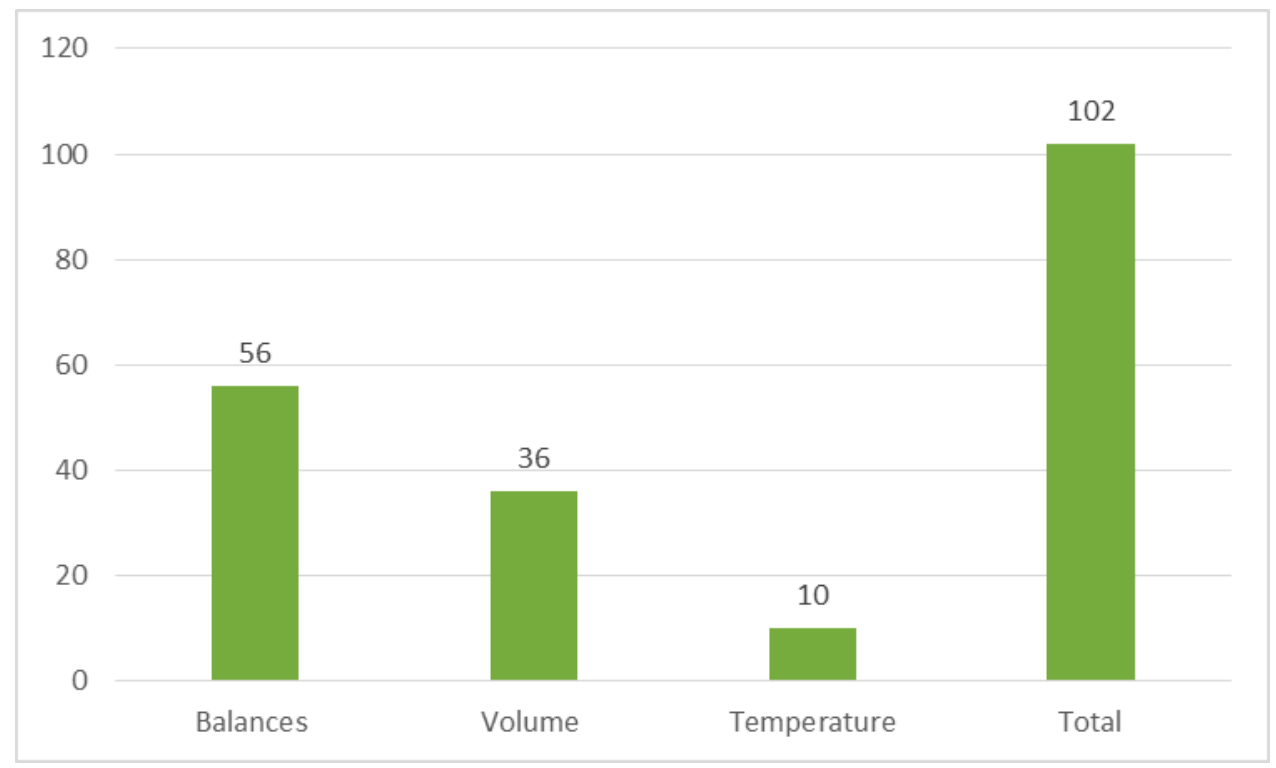

Fig. 2. Number of students rated as calibrator for each magnitude 


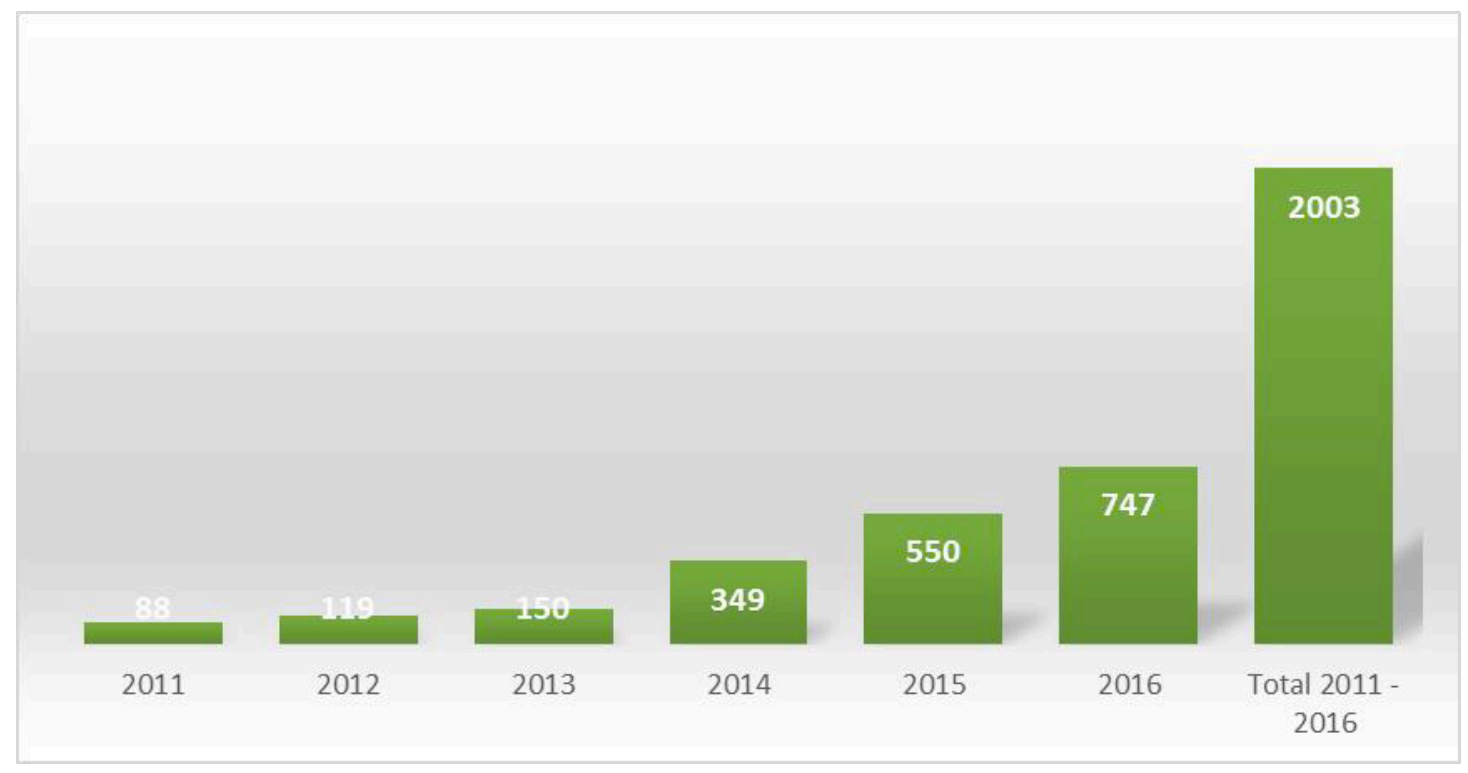

Fig. 3. Number of calibrations performed for years in the period $2011-2016$

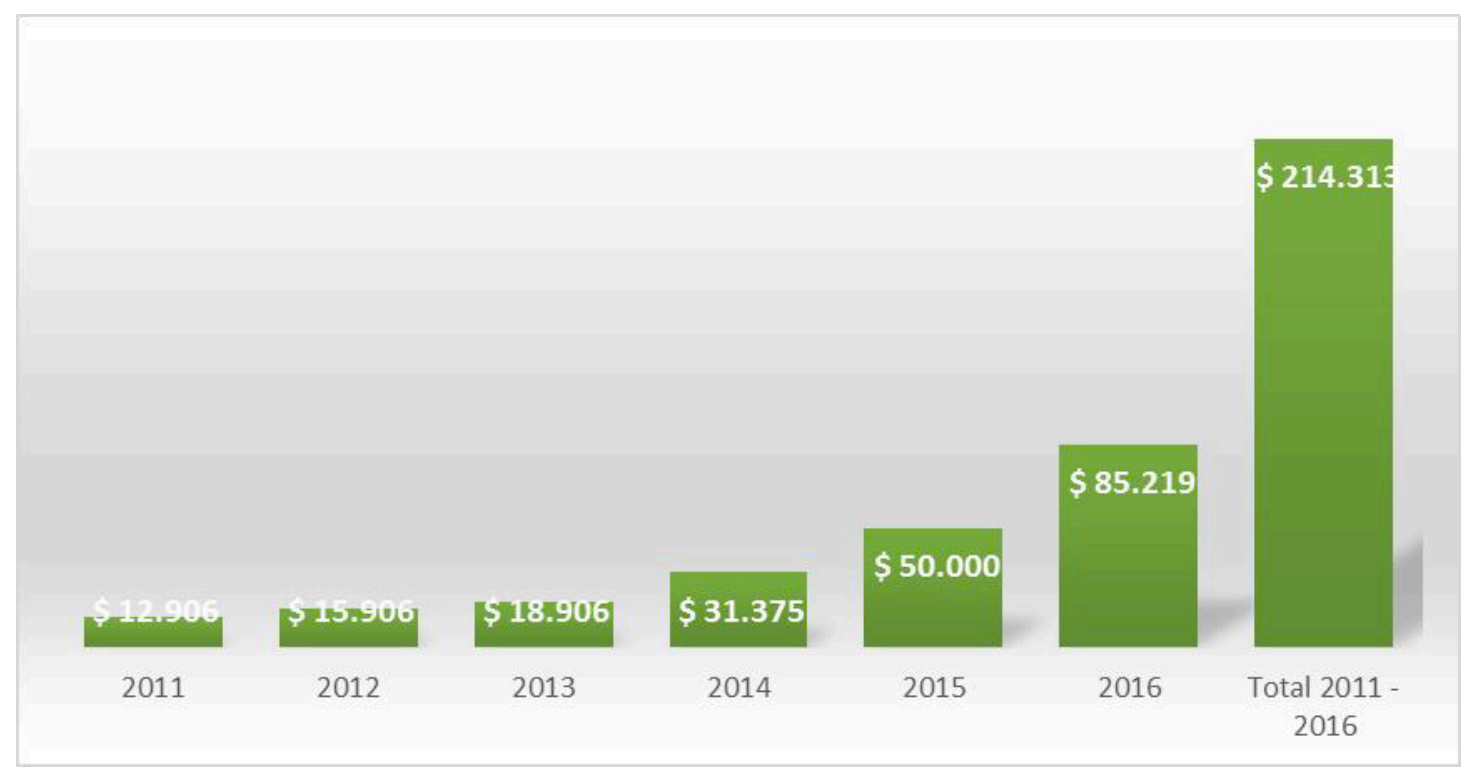

Fig. 4. Government budget savings for in-house calibrations in dollars per year and total in the period 2011-2016 


\section{CONCLUSIONS}

Metrology wasn't a popular matter at INTA. After the formation and training started, more agents were aware of its importance and relevance in INTA. New agents have been trained and new magnitudes have been included in SGM when the needs were detected and the commitment with the accuracy for results was appreciated.

The formation and training in Metrology has allowed to have authorized staff to calibrate in different magnitudes in house in different agencies, reducing the costs.

The number of services given by SGM has increased year to year; a trained agent with the equipment available performed each calibration using the software design also in house. The centralized training and in house metrological services in an organization with the size and complexity of INTA probably has allowed to generate economies of scale, organizational culture and a common language. The evolutionary collective learning and exchange of experiences has possibly facilitated the services of the SGM in the several agencies and resulted in lower costs.

\section{REFERENCES}

ISO 9001:2015 Quality Management Systems. Requirements ISO/IEC 17025:2005. General requirements for the competence of testing and calibration laboratories INTA. 2004. PEI 2005-2015. EI INTA que queremos. Plan Estratégico Institucional. 2005 - 2015. Slepetis, C. 2011. Formación en Calidad. EI Caso INTA. Comunicaciones. VI IBEROLAB. QMS documentes. Gerencia de Procesos y Calidad. INTA. 